\title{
The Transcription Factor Nuclear Factor of Activated T Cells c3 Modulates the Function of Macrophages in Sepsis
}

\author{
Ravi Ranjan $^{\mathrm{a}}$ Jing Deng ${ }^{\mathrm{b}}$ Sangwoon Chung ${ }^{\mathrm{b}}$ Yong Gyu Lee ${ }^{\mathrm{b}}$ \\ Gye Young Park $^{\mathrm{a}}$ Lei Xiao $^{\mathrm{a}}$ Myungsoo Joo $^{\mathrm{c}}$ John William Christman ${ }^{\mathrm{b}}$ \\ Manjula Karpurapu ${ }^{\mathrm{b}}$ \\ ${ }^{a}$ Department of Medicine and Section of Pulmonary, Critical Care, Sleep and Allergy, Department of Medicine, \\ University of Illinois, Chicago, Ill., and b Section of Pulmonary, Allergy, Critical Care and Sleep Medicine, The \\ Ohio State University Wexner Medical Center, Davis Heart and Lung Research Institute, Columbus, Ohio, USA; \\ 'Department of Immunology, Pusan University, Pusan, Republic of Korea
}

\section{Key Words}

Inducible nitric oxide synthase · Macrophages - Nuclear

factor of activated T cells · Sepsis · VIVIT

\begin{abstract}
The role of the transcription factor nuclear factor of activated T cells (NFAT) was initially identified in T and B cell gene expression, but its role in regulating gene expression in macrophages during sepsis is not known. Our data show that NFATC3 regulates expression of inducible nitric oxide synthase (iNOS) in macrophages stimulated with lipopolysaccharide. Selective inhibition of NFAT by cyclosporine A and a competitive peptide inhibitor 11R-VIVIT inhibited endotoxin-induced expression of iNOS and nitric oxide (NO) release. Macrophages from NFATc3 knockout (KO) mice show reduced iNOS expression and NO release and attenuated bactericidal activity. Gel shift and chromatin immunoprecipitation assays show that endotoxin challenge increases NFATC3 binding to the iNOS promoter, resulting in transcriptional activation of iNOS. The binding of NFATC3 to the
\end{abstract}

iNOS promoter is abolished by NFAT inhibitors. NFATc3 KO mice subjected to sepsis show that NFATc3 is necessary for bacterial clearance in mouse lungs during sepsis. Our study demonstrates for the first time that NFATc3 is necessary for macrophage iNOS expression during sepsis, which is essential for containment of bacterial infections.

(c) 2014 S. Karger AG, Basel

\section{Introduction}

Severe sepsis is a common medical condition characterized by a systemic inflammatory response that results from a harmful or damaging host response to infection that leads to multiple organ failure and death $[1,2]$. It is imperative to improve the understanding of the dysregulation of the host response/interactions during sepsis, which would lead to new opportunities for therapeutic intervention $[3,4]$. Macrophages have a major role in the maintenance of the tissue homeostasis, in part because of antimicrobial properties and through involvement in

\section{KARGER}

(C) 2014 S. Karger AG, Basel

1662-811X/14/0066-0754\$39.50/0

E-Mail karger@karger.com

www.karger.com/jin
Dr. Manjula Karpurapu

Section of Pulmonary Allergy, Critical Care and Sleep Medicine

The Ohio State University Wexner Medical Center

Davis Heart and Lung Research Institute, Columbus, OH 43210 (USA)

E-Mail Manjula.Karpurapu@ osumc.edu 
both the innate and adaptive immune responses during the course of inflammatory diseases. Macrophages are innate immune effector cells that are essential for generating beneficial inflammation that is necessary for pulmonary antibacterial host defense $[2,5]$. Severe sepsis that is associated with Gram-negative bacteremia is characterized by neutrophilic lung inflammation and pulmonary vascular hyperpermeability, which are characteristic of the acute respiratory distress syndrome [3]. Macrophages express high levels of inducible nitric oxide synthase (iNOS) and are important sources of iNOS-derived nitric oxide (NO), which together with the respiratory burst of reactive oxygen species (ROS) leads to effective bactericidal activity $[2,6]$. NO has a paradoxical role in severe sepsis, where it has a harmful effect by contributing to lung injury in animals and humans $[7,8]$ and a beneficial effect on the immune system and in the lungs through antimicrobial activity [9-11]. Recently it has been reported that monocyte/macrophage-derived iNOS plays a pivotal role in mediating resolution of lung injury by modulating lung immune responses, thus facilitating clearance of alveolar inflammation and promoting lung repair [12]. Activation of macrophages is a key component of the immune response, and several bacterial products and proinflammatory cytokines participate actively in triggering this process. Activation of macrophages is dependent on the Toll-like receptor (TLR) signaling pathway, which engages transcriptional regulators to induce proinflammatory genes for antimicrobial activity [13]. However, uncontrolled or excess proinflammatory mediators and ROS can exert deleterious effects such as those observed during severe sepsis and in persistent local inflammatory processes [14]. Thus it is important to understand the potential role of transcription factors that regulate the gene expression in macrophages and immune cells.

Nuclear factors of activated T cells (NFATs) are transcription factors that tightly control proinflammatory cytokine expression for adaptive immunity in $\mathrm{T}$ and $\mathrm{B}$ lymphocytes. However, little is known about the role of NFATs for innate immunity in macrophages. The NFAT gene family consists of 5 members; NFATc1-c4 are controlled by calcium levels, while NFAT5 is activated by osmotic stress $[15,16]$. Calcium mobilization through $\mathrm{Ca}^{2+}$ release-activated $\mathrm{Ca}^{2+}$ channels has been observed in macrophages in response to bacterial infection $[17,18]$. Recently it has been reported that the calcium entry controller channel transient receptor potential melastatin- 4 mobilizes calcium entry and maintains $\mathrm{Ca}^{2+}$ homoeostasis, which specifically affects the functions of macro- phages in sepsis [19]. An increase in intracellular calcium levels causes calcineurin, a calcium-dependent phosphatase, to dephosphorylate cytoplasmic NFAT, resulting in its activation and translocation to the nucleus and ensuing transcription of the target genes. Activated NFAT is negatively regulated by rephosphorylation activity of various nuclear NFAT kinases and exported back to the cytoplasm [15]. NFATc3 and NFATc4 are involved in the TLR-activated innate inflammatory response and cytokine induction [20]. In neurons, NFATc3 undergoes rapid dephosphorylation and nuclear translocation, whereas NFATc4 remains phosphorylated and localized to the cytosol [21]. Recently it has been reported that blocking NFAT function is beneficial in inflammatory diseases like inflammatory bowel disease and pulmonary arterial hypertension $[22,23]$, which suggests that NFAT may alter NO production. However, the molecular mechanisms and physiological relevance underlying the transcription regulation of iNOS in macrophages by NFAT have not been previously reported in the context of bactericidal activity.

In this study, we demonstrate that the transcription factor NFATc3 mediates the expression of iNOS in macrophages and that abrogation of NFAT results in unrestrained polymicrobial sepsis and inflammation in mice lungs.

\section{Materials and Methods}

\section{Mouse Strains Used in This Study}

$\mathrm{NFATc}^{-/-}$[NFATc3 knockout (KO)] and wild-type (WT) C57BL/6J $\left(\mathrm{NFATc}^{+/+}\right)$mice were purchased from The Jackson Laboratory. NFATc ${ }^{-/-}$and WT mice 8-12 weeks of age were used in the study, and all the experiments were conducted with protocols approved by the Institutional Animal Care and Use Committee of The Ohio State University, Columbus, Ohio, and the University of Illinois, Chicago, Ill., USA.

\section{Culture of Immortalized Mouse Bone Marrow-Derived}

Macrophages

Immortalized mouse bone marrow-derived macrophages generated from transgenic luciferase-expressing HLL mice (BMDMLuc) were generated as described previously [24] and were cultured in complete medium [1× DMEM containing 10\% FBS (HyClone), supplemented with penicillin $(100 \mathrm{U} / \mathrm{ml}) /$ streptomycin $(100 \mu \mathrm{g} / \mathrm{ml}$; Invitrogen) $]$ and maintained at $37^{\circ} \mathrm{C}$ in humidified air containing $5 \% \mathrm{CO}_{2}$.

\section{Isolation and Culture of BMDM}

Femoral and tibia bone marrow was isolated from mice (WT, NFATc3 KO and HLL) as previously described [25]. Mouse bone marrow cells were flushed from femurs and tibias with $\mathrm{Ca}^{2+} / \mathrm{Mg}^{2+}$ free Hanks' balanced salt solution (Mediatech Inc.). Bone marrow 
progenitor cells were cultured in DMEM (Mediatech) containing $10 \%$ heat-inactivated FBS and $100 \mathrm{U}$ of penicillin-streptomycin (Mediatech) and supplemented with 10\% L929 Cell Culture Media. After 7 days, BMDM (approx. 99\% macrophages based on flow cytometry using anti-F4/80) were collected for the experiments.

\section{Expression of iNOS in Mouse Macrophages}

BMDM-Luc were seeded in tissue culture dishes in complete medium, grown for $24 \mathrm{~h}$ and starved in $2 \% \mathrm{FBS}$-containing medium overnight. The cells were treated with $100 \mathrm{ng} / \mathrm{ml}$ lipopolysaccharide (LPS) Escherichia coli serotype R515, TLR grade (581-007L002, Enzo Life Sciences), for various periods of time (0, 2, 4, 8, 16 and $24 \mathrm{~h}$ ). The expression of iNOS was analyzed by reverse transcription quantitative real-time PCR (RT-qPCR) and Western blot.

\section{Inhibition of iNOS Expression in Mouse Macrophages}

Inhibition of iNOS expression in macrophage cells was examined by using the NFAT-specific inhibitors cyclosporine A (CsA; Enzo Life Sciences) and the cell-permeable peptide 11R-VIVIT (Calbiochem). 11R-VEET custom synthesized by GenScript was used as control peptide. The BMDM-Luc were seeded in culture dishes in complete medium for $24 \mathrm{~h}$ and then starved in 2\% FBScontaining medium overnight. Subsequently, cells were treated with CsA $(10 \mu \mathrm{M}, 30 \mathrm{~min})$ or 11R-VIVIT $(5 \mu \mathrm{M}, 1 \mathrm{~h})$ and then challenged with LPS (100 ng/ml) for 12-16 h. The cells were lysed in radioimmunoprecipitation assay buffer and immunoblotted for iNOS.

\section{Measurement of Nitrite in Macrophage Culture Media}

BMDM-Luc and BMDM were treated either with LPS (100 ng/ $\mathrm{ml})$ alone or with CsA $(10 \mu \mathrm{M}), 11 \mathrm{R}-\mathrm{VEET}(50 \mu \mathrm{M})$ or $11 \mathrm{R}-\mathrm{VIVIT}$ $(50 \mu \mathrm{M})$ for the indicated time periods. Culture supernatants were stored at $-70^{\circ} \mathrm{C}$ for analysis of nitrite. Nitrite concentration was determined in culture supernatant with a Griess Reagent Kit (G7921, Molecular Probes Inc.) in a 96-well microplate assay system according to the manufacturer's protocol. The nitrite concentration in the samples was calculated from a standard curve of sodium nitrite.

\section{Reverse Transcription Quantitative Real-Time PCR}

Total RNA was isolated using the RNeasyPlus Mini kit (Qiagen) according to the manufacturer's protocol, and the quality was assessed by agarose gel electrophoresis. Purified total RNA $(2.5 \mu \mathrm{g})$ was reverse transcribed using the RevertAid First Strand cDNA Synthesis kit (Thermo Scientific) with random hexamer primers. The $i N O S$ and $\beta$-actin mRNA levels were determined by RT-qPCR on a LightCycler 480 real-time PCR instrument using LightCycler 480 SYBR Green I Master reagent, and $2 \mu$ of the cDNA was used with the following conditions: initial denaturation at $95^{\circ} \mathrm{C}$ for 10 min, and a loop of 45 cycles consisting of denaturation at $95^{\circ} \mathrm{C}$ for $15 \mathrm{~s}$, annealing at $68^{\circ} \mathrm{C}$ for $30 \mathrm{~s}$ and extension at $68^{\circ} \mathrm{C}$ for $30 \mathrm{~s}$. The results were analyzed with LightCycler 480 Software 1.5.0. by an advanced relative quantification algorithm (Roche Diagnostics). Sequences of murine iNOS primers are as follows: miNOS2F, 5'-CCG CCC TGG TGC AGG GAA TC-3', and miNOS2R, $5^{\prime}$-ACC CAG TAG CTG CCG CTC TCA-3'. For murine $\beta$-actin, primers are mActinF $\left(5^{\prime}\right.$-GTC GAG TCG CGT CCA CCC GC- $\left.3^{\prime}\right)$ and $\mathrm{mActinR}\left(5^{\prime}\right.$-CGT CAT CCA TGG CGA ACT GGT GGC-3').

\section{Activation of NFATc3 during LPS Stimulation}

The BMDM from WT mice were treated with LPS $(100 \mathrm{ng} / \mathrm{ml})$ for $0,0.5$ and $4 \mathrm{~h}$ and washed in cold $1 \times$ PBS. The cellular and nuclear protein fractions were separated using a Pierce NE-PER Nuclear and Cytoplasmic Extraction Reagents kit (catalogue No. 78833), resolved on 10\% SDS-polyacrylamide gels and immunoblotted with different NFAT antibodies. The antibodies used for immunoblotting were NFATc1 (8032S, Cell Signaling), NFATc2 (4389S, Cell Signaling), NFATc3 (4998S, Cell Signaling) and NFATc4 (sc-13036, Santa Cruz Biotechnology Inc.). Purity of cytoplasmic and nuclear preparations was assessed by $\beta$-actin and p53 (sc-6243, Santa Cruz Biotechnology).

\section{Western Blot}

After appropriate treatment, BMDM-Luc were lysed with $1 \times$ radioimmunoprecipitation assay buffer [ $50 \mathrm{~mm}$ Tris- $\mathrm{HCl}, \mathrm{pH} 8.0$, $150 \mathrm{mM}$ sodium chloride, 1.0\% Igepal CA-630 (NP-40), 0.5\% sodium deoxycholate and $0.1 \%$ sodium dodecyl sulfate] containing $1 \times$ protease inhibitor cocktail (Roche Diagnostics). Cell lysates containing equal amounts of protein were fractionated by electrophoresis on a reducing polyacrylamide gel and electroblotted to a nitrocellulose membrane (BioRad). The nitrocellulose membranes were incubated with blocking buffer [50 mM Tris-HCl, pH 7.4, 150 $\mathrm{mM} \mathrm{NaCl}, 0.1 \%$ Tween-20 (TBST) containing 5\% BSA] for $1 \mathrm{~h}$ at room temperature, followed by overnight incubation with primary antibody at $4^{\circ} \mathrm{C}$ in blocking buffer and then with a horseradish peroxidase-conjugated secondary antibody. After washing the membrane with TBST, the reactive bands were detected by enhanced chemiluminescent substrate (SuperSignal West Pico, Thermo Scientific). Antibodies used were anti-iNOS/NOS Type II (610332, BD Transduction Laboratories), anti- $\alpha$-tubulin (sc-8035, Santa Cruz Biotechnology) and anti- $\beta$-actin (Sigma).

\section{Analysis of iNOS Promoter}

The genomic sequence containing the promoter region of mouse $i N O S$ mRNA was downloaded from the National Center for Biotechnology Information database. Potential NFAT binding sites were identified by analysis of the promoter region using the online software Patch 1.0 (www.gene-regulation.com/pub/programs.html) and MatInspector (www.genomatix.de) [26].

\section{Electrophoretic Mobility Shift Assay}

BMDM-Luc were grown in complete medium, serum starved overnight, treated with CsA $(10 \mu \mathrm{M})$ and then stimulated with LPS $(0.1 \mu \mathrm{g} / \mathrm{ml})$ for 30 and $60 \mathrm{~min}$. The cells were washed with ice-cold $1 \times$ PBS and lysed with Buffer A [10 mM HEPES, pH 7.9, $1.5 \mathrm{~mm}$ $\mathrm{MgCl}_{2}, 10 \mathrm{mM} \mathrm{KCl}, 0.1 \%$ Triton-X-100 and protease inhibitor cocktail (Roche)] for $15 \mathrm{~min}$ at $4^{\circ} \mathrm{C}$. The content was centrifuged, and the pellet was washed with Buffer B [10 mM HEPES, pH 7.9, $1.5 \mathrm{mM} \mathrm{MgCl}_{2}, 10 \mathrm{mM} \mathrm{KCl}$ and protease inhibitor cocktail (Roche)]. The supernatant was discarded, and the nuclear pellet was lysed with Buffer C [20 mM HEPES, pH 7.9, $1.5 \mathrm{mM} \mathrm{MgCl}_{2}, 420 \mathrm{mM}$ $\mathrm{NaCl}, 0.2$ mM EDTA, pH 8, 25\% glycerol, 1 mM DTT and protease inhibitor cocktail (Roche)]. The NFAT consensus DNA probes were obtained from Santa Cruz Biotechnology (catalogue No. sc2577) and labeled using the Biotin Labeling kit (catalogue No. 89818, Thermo Scientific). Equal amounts of protein from different treatments were incubated with a biotin-labeled NFAT consensus probe and resolved on $5 \%$ polyacrylamide native gel in $0.5 \times$ Tris-borate-EDTA buffer. The protein-DNA complexes were 
transferred to Biodyne A membranes (catalogue No. 77015, Thermo Scientific). For super shift assays, the nuclear extract was incubated with anti-NFATc3, and for controls, the respective IgGs were used. For the electrophoretic mobility shift (EMSA) competition assay, $5 \mu \mathrm{g}$ of nuclear extract was incubated with $10 \mathrm{pmol}$ of biotin-labeled NFAT consensus oligo using reaction buffer supplied in the Pierce LightShift EMSA kit (Thermo Scientific, No. 20148). In the cold competition assay, unlabeled NFAT consensus oligo was included in excess at a concentration of 10,30 or $50 \mathrm{pmol}$ separately in the above reaction mix and analyzed. The reaction products were resolved on $5 \%$ polyacrylamide native gel in $1 \times$ Tris-borate-EDTA buffer and transferred onto a Pierce Biodyne Nylon membrane. The biotinylated products were detected using the Chemiluminescent Nucleic Acid Detection Module (Thermo Scientific) on the Gel Imaging System (BioRad).

\section{Chromatin Immunoprecipitation}

BMDM-Luc were treated as described above. The cells were washed twice with $1 \times$ PBS, and proteins were cross-linked with $1 \%$ formaldehyde (Electron Microscopy Sciences) for $10 \mathrm{~min}$ at room temperature. The reaction was quenched by adding $125 \mathrm{~mm}$ glycine for $5 \mathrm{~min}$, and cells were washed twice with $1 \times$ PBS and stored at $-80^{\circ} \mathrm{C}$. Chromatin was extracted using the ChromaFlash Chromatin extraction kit (Epigentek Group Inc.), and the chromatin was sheared by using a sonicator (Virtis Virsonic Cell Disrupter) for $5 \mathrm{~min}$, with a 10-second pulse on, and a gap of $20 \mathrm{~s}$, and was repeated 3 times. Chromatin immunoprecipitation (ChIP) was carried out using the ChromaFlash One-Step ChIP kit (Epigentek) with the manufacturer's recommended protocol. The DNA-protein complex was immunoprecipitated using a mixture of anti-NFATc3 antibodies (sc-23814 and sc-1152, Santa Cruz Biotechnology). An unrelated goat polyclonal antibody (anti-GFP, sc-5385) and anti-RNA Polymerase II (sc-5943) were used as controls. The immunoprecipitated DNA fragments were analyzed by RT-qPCR using PerfeCTa SYBR Green FastMix (Quanta BioSciences Inc.) containing $250 \mathrm{nM}$ of each primer (forward: 5'-CTG TTC CCC TTA GGT GGG-3'; reverse: $5^{\prime}$-ACA GGA GTG TGT GCA TCT G-3') with initial denaturation at $95^{\circ} \mathrm{C}$ for $5 \mathrm{~min}$ and a loop of 45 cycles consisting of denaturation at $95^{\circ} \mathrm{C}$ for $15 \mathrm{~s}$, annealing at $55^{\circ} \mathrm{C}$ for $30 \mathrm{~s}$ and extension at $72^{\circ} \mathrm{C}$ for $45 \mathrm{~s}$, on a LightCycler 480 real-time PCR instrument (Roche Diagnostics). The crossing point values were analyzed with LightCycler 480 Software 1.5.0 SP4. The abundance of NFATc3 on the iNOS promoter or the fold enrichment was calculated using the equation mentioned in the ChromaFlash One-Step ChIP kit (Epigentek). The unprecipitated chromatin was used as input control for DNA and used for RTqPCR. An unrelated DNA sequence (approx. $3 \mathrm{~kb}$ downstream of the start codon of iNOS) was analyzed for absence of NFAT binding sites by MatInspector, and primers were designed for control ChiP experiments (forward: $5^{\prime}$-AGG GTC ACT TCC CTC AGT AT-3'; reverse: $5^{\prime}$-CAC TCT GCC TGT GTC TTA TCT T-3').

Synthesis of the Control Peptide 11R-VEET

The control peptide, 11R-VEET [23] (RRRRRRRRRRRGGGMAGPPHIVEETGPHVI; scrambled sequence of 11R-VIVIT), was custom synthesized and HPLC-purified (95.0\% purity) by GenScript (Piscataway, N.J., USA).

In vitro Bactericidal Assay of Macrophages Using E. coli

The in vitro bactericidal assay was performed according to a previously published protocol with slight modifications [27].
BMDM were cultured from WT and NFATc3 KO mice in 12-well tissue culture plates starved of serum overnight in DMEM containing 2\% FBS. Overnight culture of E. coli DH5a (Invitrogen Corporation) was used to assess the bactericidal activity of macrophages. Bacterial cell number was determined by measuring the optical density at $600 \mathrm{~nm}$. In the first set of experiments, the WT BMDM were treated with the control peptide 11R-VEET or the NFAT inhibitor 11R-VIVIT and exposed to bacteria. In the second set, the BMDM from WT and NFATc3 KO mice were exposed to bacteria. E. coli $\mathrm{DH} 5 a$ was added to each well containing the macrophages, and after 90 min, cells were washed with DMEM to get rid of the nonphagocytized bacteria, and the medium was replenished with DMEM containing 2\% FBS and incubated overnight. The macrophages were lysed with Milli-Q water, plated on Luria Bertani agar culture plates and incubated at $37^{\circ} \mathrm{C}$. Colony-forming units were enumerated with the Colony Counter program using Quantity One Software (BioRad).

\section{Polymicrobial Infection Assay in Lungs}

The WT and NFATc3 KO mice were subjected to sepsis by the cecal ligation and puncture method (CLP) [28]. In this method, a midline incision of about $1 \mathrm{~cm}$ was made in the lower abdominal area, and the cecum was exposed, ligated with a sterile silk thread and punctured twice with a 21-gauge needle. After puncturing, a very small amount of fecal material was allowed to extrude into the abdominal cavity. The viscera and skin layers were sutured back in place with a 6.0 Nylon silk suture. In sham controls, the cecum was exposed by opening the visceral cavity but was neither ligated nor punctured. The animals were resuscitated with $0.5 \mathrm{ml}$ of saline subcutaneously. Mice were maintained in aseptic conditions, and $24 \mathrm{~h}$ after surgery (sham and CLP), the mice were euthanized and lungs were surgically excised, minced in DMEM and ground using a handheld mechanical homogenizer. The contents were spun, and $100 \mu \mathrm{l}$ of the tissue supernatant was spread on tryptic soy agar sheep blood agar culture plates (Remel Products, Thermo Fisher Scientific) and incubated at $37^{\circ} \mathrm{C}$. Colony-forming units were enumerated with the Colony Counter program using Quantity One Software (BioRad).

\section{Statistical Analysis}

Statistical analyses were performed using a nonpaired Student's t test, and $\mathrm{p} \leq 0.05$ was considered significant.

\section{Results}

\section{Transcription Factor NFATc3 Mediates Expression of} iNOS in Macrophages

BMDM-Luc were used to analyze the time course for expression of iNOS in response to LPS $(100 \mathrm{ng} / \mathrm{ml})$. The expression of $i N O S$ mRNA and protein peaked approximately $8-16$ h after LPS treatment (fig. 1a,b), and this time point was used for subsequent experiments. The transcription factor NFAT has been shown to play an important role in the regulation of cytokine expression for adaptive immunity in $\mathrm{T}$ and B lymphocytes, and we hypothesized that NFAT plays an essential role in the expression 


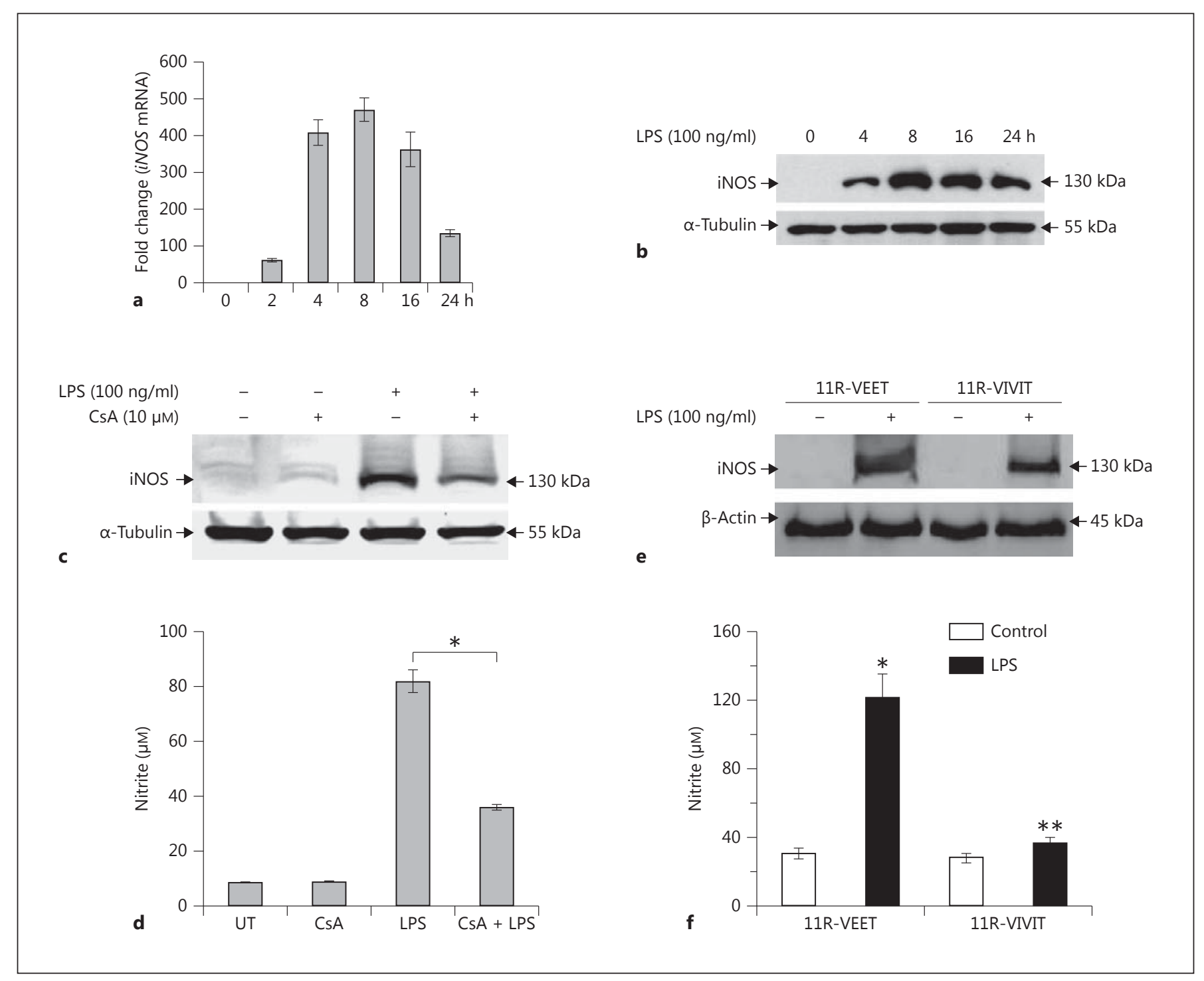

Fig. 1. Expression of $i N O S$ in macrophages is regulated by NFAT. BMDM-Luc were treated with LPS (100 ng/ml), and expression of iNOS was determined at various time points. a, b iNOS mRNA levels were determined by RT-qPCR (a), and protein level was determined by Western blot (b). Expression peaked at approximately 8-16 h following treatment with LPS. c, e Macrophages pretreated with specific inhibitors of NFAT (CsA and 11R-VIVIT, respectively) and challenged with LPS showed significant inhibition of expression of iNOS (c, e, representative Western blots). d, $\mathbf{f}$ The concentration of nitrite in the cell culture media, which is an indicator of iNOS expression, was also measured. The concentration of nitrite in the presence of NFAT inhibitors CsA (d) and $11 R-V I V I T(\mathbf{f})$ was analyzed. Treatment with specific NFAT inhibitors resulted in significant reduction of nitrite in cell culture media, indicating that the expression of iNOS was reduced. 11RVEET was used as control. The error bars represent the SEM from 3 independent experiments. ${ }^{*} \mathrm{p} \leq 0.05,{ }^{* *} \mathrm{p} \leq 0.005$. UT $=\mathrm{Un}-$ treated. of iNOS in macrophages. To address this hypothesis, we initially used 2 specific inhibitors of NFAT, CsA and a cell membrane-permeable peptide (11R-VIVIT), to determine the impact on expression of iNOS in endotoxin (LPS)-stimulated BMDM-Luc. CsA inhibits the activity of calcinuerin phosphatase of proteins including NFAT [29], whereas the cell-permeable 11R-VIVIT inhibits NFAT activation through interaction with the calcineurin binding site for NFAT and thus prevents nuclear translocation without affecting calcineurin phosphatase activity [30]. As shown, the expression of iNOS in the NFAT inhibitortreated BMDM-Luc was decreased by more than $50 \%$ 


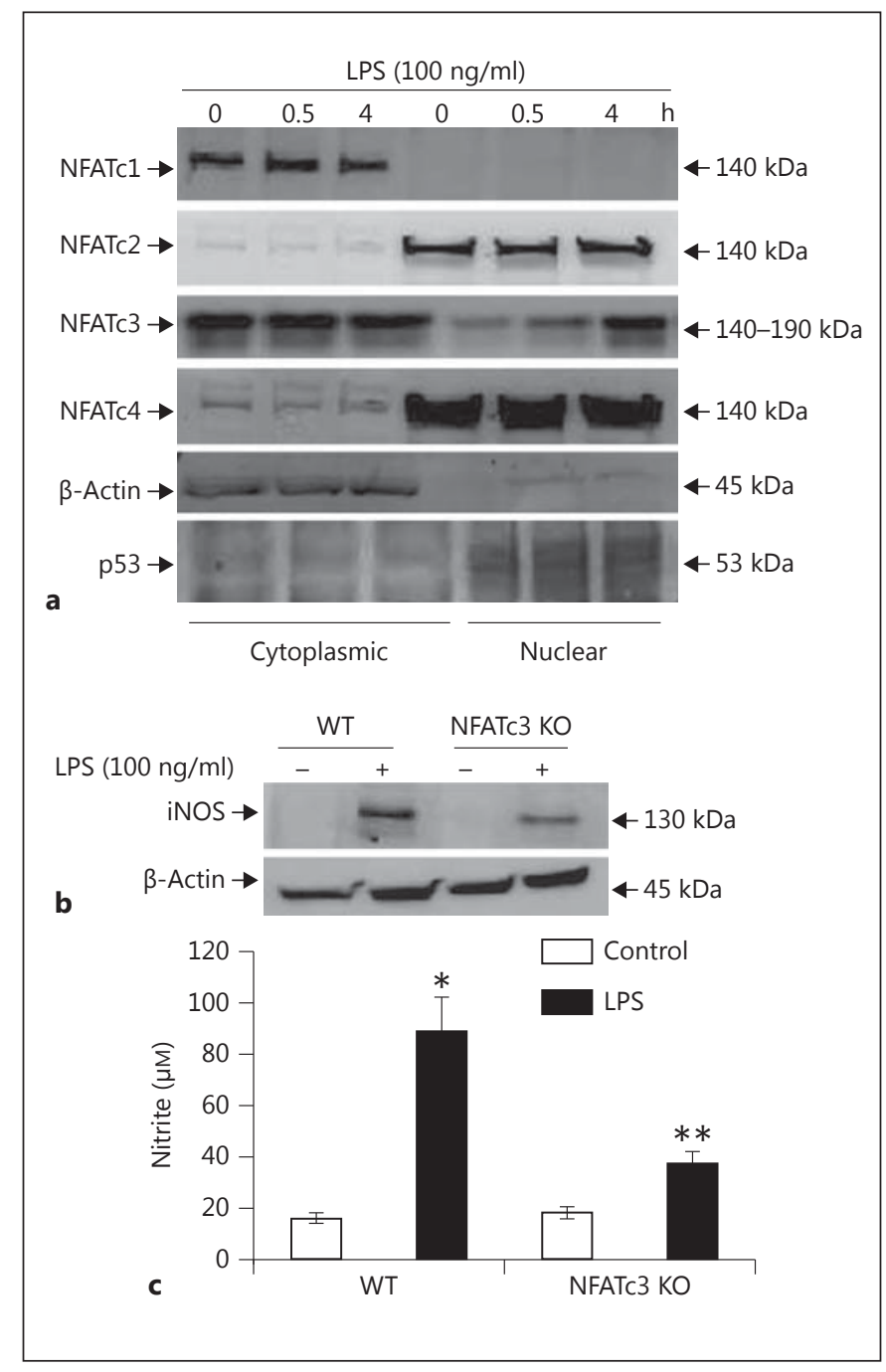

Fig. 2. LPS causes NFATc3 nuclear translocation specifically, which regulates the iNOS expression. a BMDM were treated with $100 \mathrm{ng} / \mathrm{ml}$ LPS for the indicated time periods, and cells were harvested. Cytosolic and nuclear protein fractions were resolved and analyzed by immunoblotting with NFATc1, NFATc2, NFATc3 and NFATc4 antibodies. b WT $\left(\mathrm{NFATc}^{+/+}\right)$and $\mathrm{NFATc}^{-/-}$ BMDM were stimulated with $100 \mathrm{ng} / \mathrm{ml} \mathrm{LPS}$ and analyzed for iNOS expression as described in figure 1 b. c WT $\left(\mathrm{NFATc}^{+/+}\right)$and $\mathrm{NFATc}^{-/-}$BMDM were stimulated with $100 \mathrm{ng} / \mathrm{ml}$ LPS and analyzed for NO release as described in figure $1 \mathrm{~d}$ and $\mathrm{f} .{ }^{*} \mathrm{p} \leq 0.05$, ** $\mathrm{p} \leq 0.005$.

when compared with the cells treated with LPS alone (fig. 1c, e). We also assessed the accumulation of nitrite $\left(\mathrm{NO}_{2}^{-}\right)$in macrophage culture media as an indicator of NO production, which is dependent on iNOS. Compared with the untreated macrophages, the accumulation of nitrite in NFAT inhibitor treatment was decreased by more than $50 \%$ (fig. 1d, f). Earlier literature indicated the role of NF- $\kappa B$ in modulating iNOS regulation $[31,32]$. To specifically identify NFAT-dependent $i N O S$ induction during sepsis, we used the NF- $\mathrm{KB}$-responsive luciferaseharboring HLL BMDM cell line. The HLL BMDM were treated with NFAT and NF- $\kappa B$ inhibitors separately, stimulated with LPS and assayed for NFAT/ NF- $\kappa B$-dependent iNOS expression/NO release and NF- $\kappa \mathrm{B}$ luciferase reporter activities. Results of the inhibitor experiments indicate that inhibition of NFAT by $11 \mathrm{R}$ VIVIT attenuates the NO release by $50-70 \%$ (online suppl. fig. 1; for all online suppl. material, see www. karger.com/doi/10.1159/000362647), whereas the NF- $\kappa B$ inhibitor decreases it by only about $30 \%$ (online suppl. fig. 1). Interestingly, NF- $\mathrm{BB}$ inhibition by QNZ decreased the NF- $\kappa \mathrm{B}$-dependent luciferase activity by more than $80 \%$, whereas VIVIT decreased it by about $40 \%$. This indicates that NO release is significantly regulated by NFAT. Together these data indicate that expression of iNOS in macrophages is significantly regulated by the transcription factor NFATc3.

\section{NFATc3 Modulates iNOS Expression at the Transcriptional Level}

The transcription factor NFAT gene family consists of 5 members; NFATc1-c4 are controlled by calcium levels, while NFAT5 is activated by osmotic stress $[15,16]$. So, to check which isoform contributed to the expression of iNOS in macrophages, we checked the nuclear translocation of the cytosolic NFAT isoforms. The WT BMDM were treated with LPS, and the nuclear and cytosolic proteins were isolated and probed for the translocation of different NFAT isoforms. Only NFATc3 translocated to the nucleus in response to LPS, compared to other members that were exclusively localized either in the cytosol or nucleus or both (fig. 2a). To further confirm that NFATc3 is actually involved in the regulation of iNOS, BMDM cultured from WT and NFATc3 KO mice were stimulated with LPS. The NFATc3 KO BMDM had significantly decreased expression of iNOS compared to WT BMDM, which also correlated with decreased NO release in culture medium (fig. 2b, c).

As LPS caused translocation of NFATc3 from the cytosol to the nucleus and associated upregulation of iNOS expression, we determined if the upregulation is mediated by binding of NFATc3 to the iNOS promoter using EMSA and ChiP assays on the iNOS promoter. In silico analysis of the genomic sequences (approx. $2 \mathrm{~kb}$ ) flanking the mouse iNOS mRNA using the online software Patch 1.0. and MatInspector revealed two putative NFAT cog- 
Fig. 3. DNA binding activity of NFATc3 is directly involved in transcriptional regulation of $i N O S$. a Schematic representation of the iNOS promoter (approx. 2-kb genomic region) representing cognate NFATc3 binding sites. b BMDM were treated with LPS and/or CsA for $30 \mathrm{~min}$; nuclear extracts were prepared as described in Materials and Methods, and an equal amount of extract was incubated with biotin-labeled doublestranded DNA containing a consensus binding site for NFAT. The NFAT proteinDNA complex was resolved on a $5 \%$ native acrylamide gel, transferred onto a Nylon membrane and developed using a Pierce chemiluminescent detection kit. c Equal amounts of nuclear extract protein were incubated with biotin-labeled double-stranded DNA containing a consensus binding site for NFAT, competing against the unlabeled (cold) probe in increasing molar concentrations and processed as described in (b). d The specificity of DNA binding was determined using NFATc3 antibody, included in the oligo-protein reaction mixture. The black arrow represents the super shift band, and white arrows represent binding activity of the NFAT probe. e, $\mathbf{f}$ Recruitment of NFATc3 to iNOS promoter was assessed by ChIP. The eluted DNA was subjected to real-time PCR, and the abundance of NFATc3 (e) and RNA Polymerase II (RNA-Pol-II; f) at the iNOS promoter was calculated using the ratio of amplification efficiency (crossing point value) of the sample over nonimmune IgG. UT $=$ Untreated.

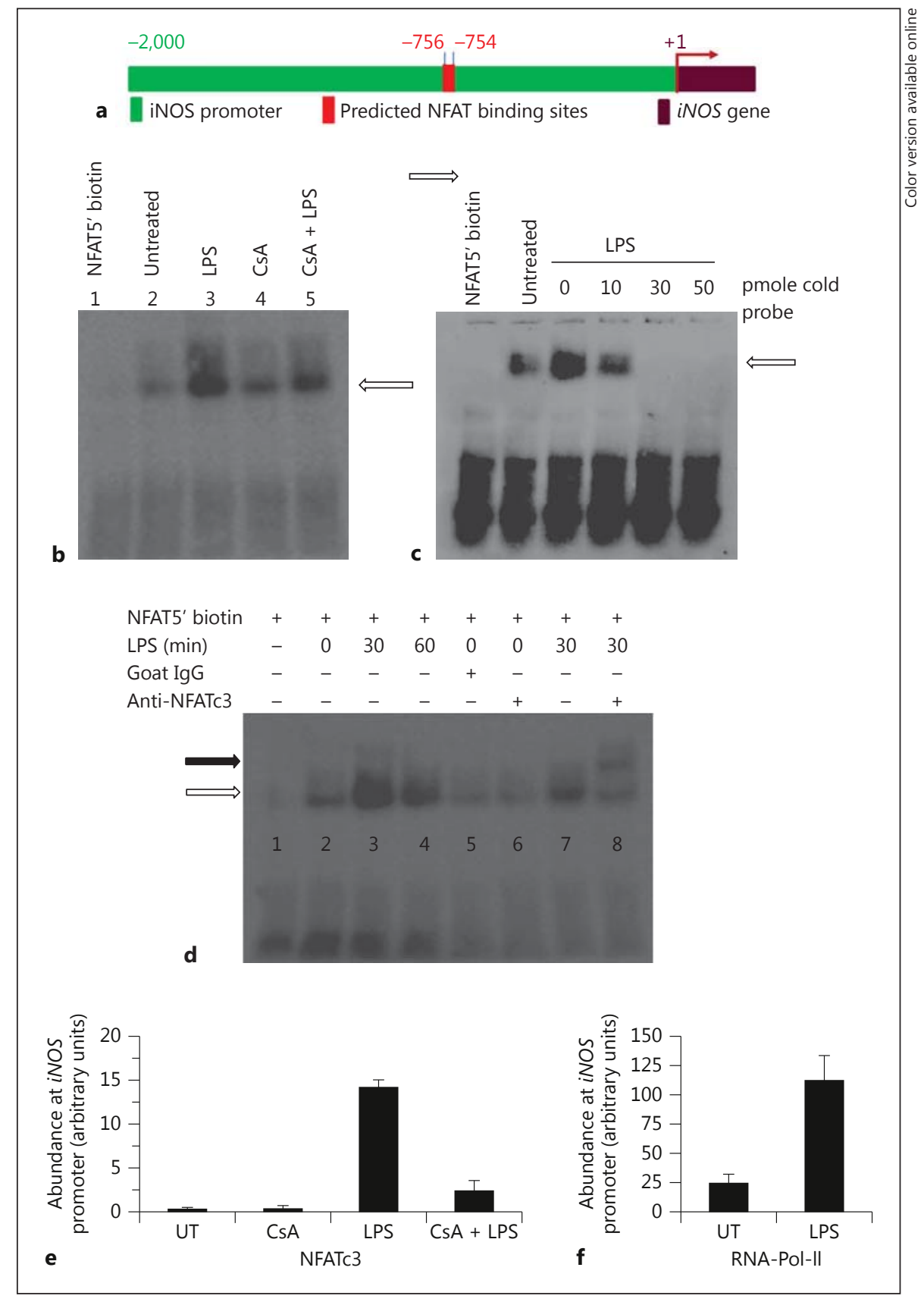

nate binding sites upstream of the start codon (ATG) at around -756 and -754 (fig. 3a). In EMSA, binding of the biotin-labeled NFAT probe with the nuclear extract was significantly reduced in BMDM pretreated with CsA (NFAT inhibitor) in response to treatment with LPS compared to LPS alone (fig. 3b, lane 3 vs. lane 5). To further confirm the specificity of DNA-protein interactions, the unlabeled (cold) probe was used in a $10-$ to $50-\mathrm{pmol}$ excess of labeled probe as competitor. The binding of the labeled probe was reduced and abolished in the presence of an increasing molar concentration of unlabeled probe (fig. 3c). The specificity of binding of the biotinylated probe with translocated NFATc3 was also confirmed using the anti-NFATc3 antibody, which caused a super shift of the DNA-protein complex (fig. 3d, lane 7 vs. lane 8). ChIP was performed to show the recruitment of NFATc3 


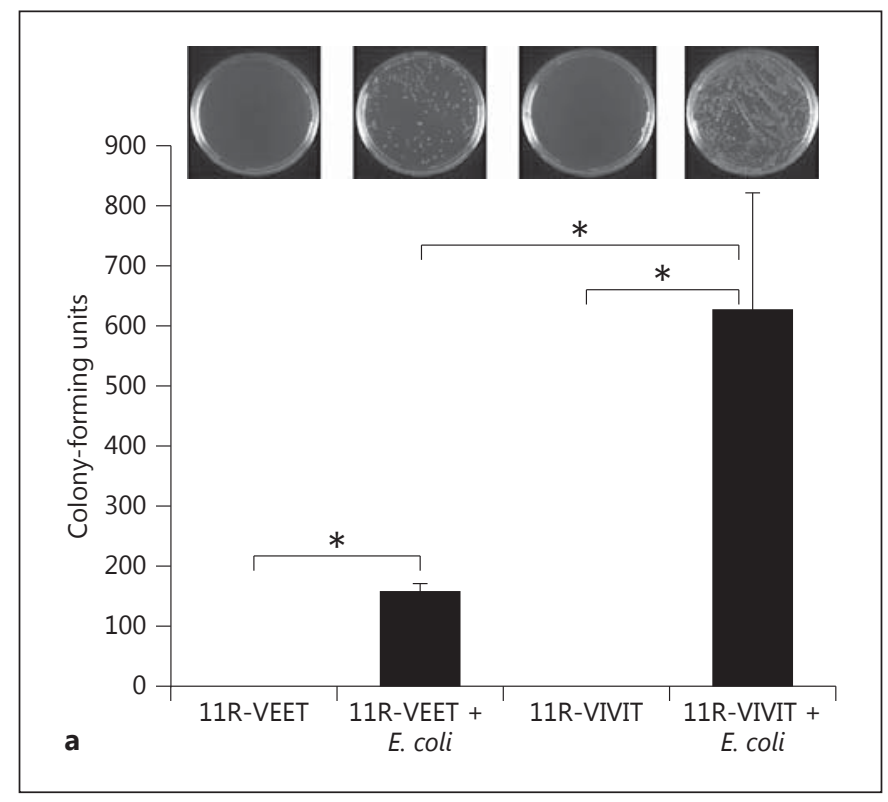

Fig. 4. Deficiency of NFATc3 results in attenuated bactericidal activity. a WT macrophages were treated with 11R-VIVIT (specific NFAT inhibitor) and incubated with E. coli, and bactericidal activity was analyzed by plating macrophage lysate on bacterial culture plates. The NFAT inhibitor-treated macrophages had attenuated bactericidal activity compared to the group treated with control

on the iNOS promoter, by designing primers encompassing putative NFAT binding sites (-756 and -754$)$. As shown, in BMDM treated with LPS, there was a high abundance of NFATc3 at the iNOS promoter, which was significantly reduced by CsA (fig. 3e); RNA Polymerase II was used as positive control for the ChIP assay (fig. 3f). We used the unprecipitated chromatin as input control for DNA, and used it for RT-qPCR, and all the reactions had similar crossing point values, indicating that an equal amount of DNA-protein complex was used for pull down (data not shown). To check the specificity of the ChIP, we designed primers from an unrelated downstream DNA sequence from the $i N O S$ gene and subjected the immunoprecipitated DNA-protein complex to RT-qPCR, and there was no significant amplification (undetected signal by machine) with the control primers, indicating the specificity of ChIP.

\section{Deficiency of NFATc3 Results in Attenuated \\ Bactericidal Activity by Macrophages}

Macrophages are inflammatory/immune effector cells that are necessary for bactericidal activity in an event of microbial infection. This activity is largely attributable to iNOS and nicotinamide adenine dinucleotide phosphate

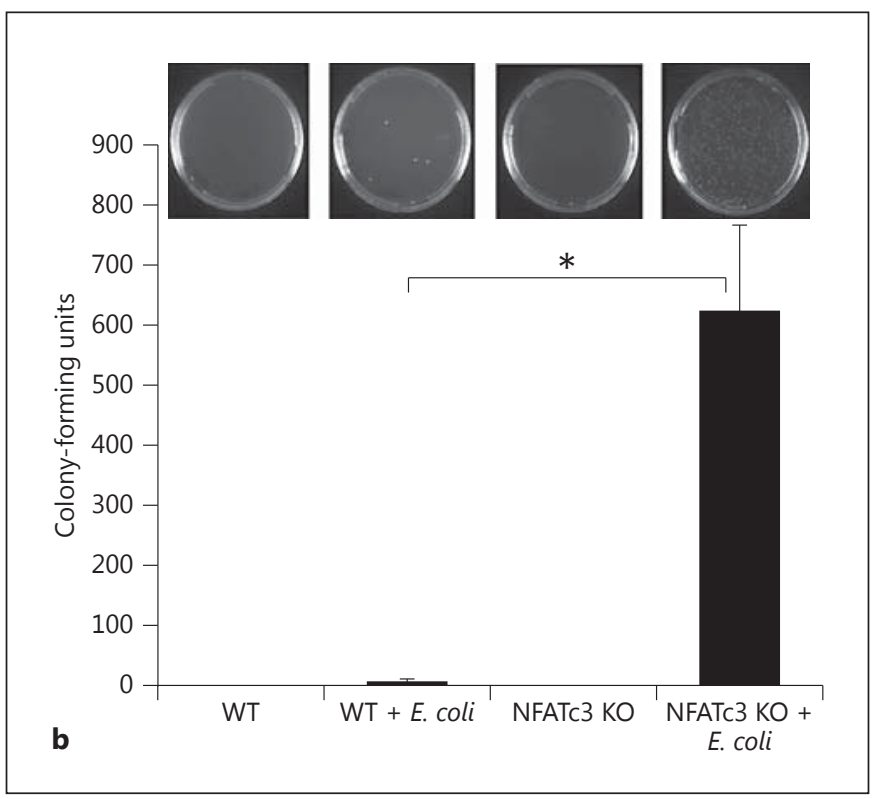

peptide (11R-VEET). b Macrophages cultured from WT and NFATc3 KO mice were treated with E. coli, and bactericidal activity was analyzed by plating macrophage lysate on bacterial culture plates. The NFATc3-deficient macrophages had significantly attenuated bactericidal activity compared to WT macrophages. Error bars represent the standard deviation. ${ }^{*} \mathrm{p}<0.01 . \mathrm{n}=3$.

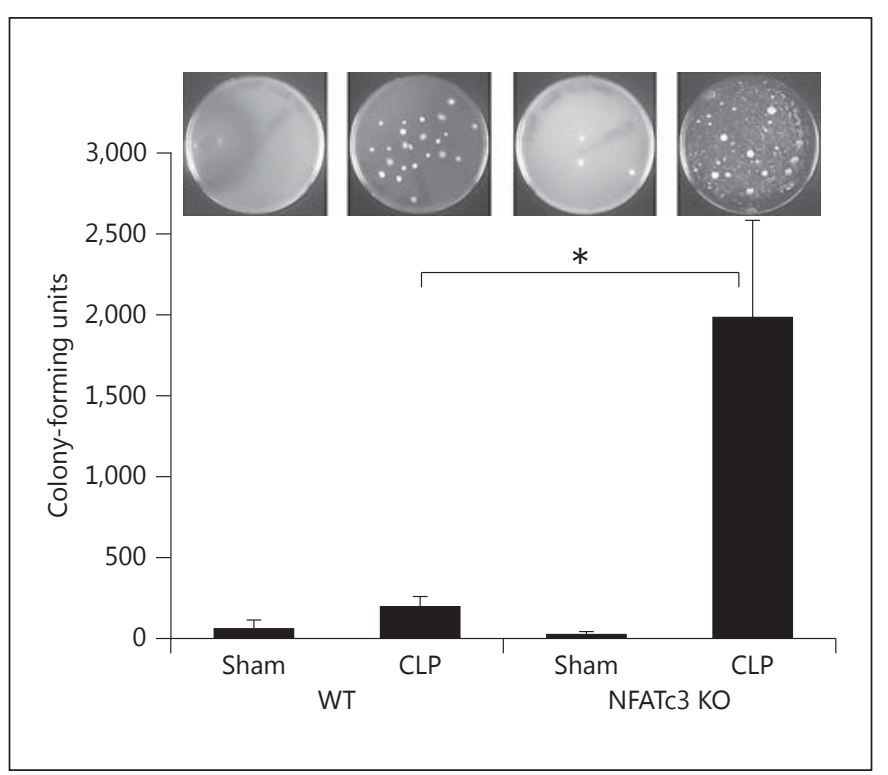

Fig. 5. Inhibition of NFATc3 results in bacterial infection in lungs during sepsis. The WT and NFATc3 KO mice were subjected to CLP. After $24 \mathrm{~h}$ the mice were euthanized, and bacterial infection in the lungs was assessed by plating lung homogenate on sheep blood agar plates. The NFATc3 KO mice subjected to sepsis by CLP had severe bacterial infection compared to WT mice. Error bars represent the standard deviation. ${ }^{*} \mathrm{p}<0.01 . \mathrm{n}=3$. 
(NADPH) oxidase, which together are the major source for reactive oxygen/nitrogen intermediates [6]. Thus, we examined the bactericidal activity of macrophages by pretreating them with a NFAT-specific inhibitor peptide (11R-VIVIT) and incubating with the bacterium E. coli. The macrophages that were treated with the inhibitor had attenuated bactericidal activity compared to control peptide (fig. 4a). In the second experiment, WT and NFATc3 KO BMDM were challenged with $E$. coli. The NFATc3 KO macrophages had attenuated bactericidal activity compared to WT macrophages (fig. 4b). These results clearly indicate that NFATc3 is required for bactericidal activity by macrophages.

\section{Inhibition of NFATc3 Results in Compromised \\ Bacterial Clearance}

To further confirm the role of NFATc3 in mediating the pathogenesis of severe sepsis, we used WT and NFATc3 KO mice. The mice were subjected to abdominal sepsis by the CLP model [28]. The NFATc3 KO mice were more susceptible to sepsis and appeared moribund compared to WT mice. The lungs from these mice were excised and assessed for bacterial clearance. The NFATc3 KO mice subjected to CLP had significant polymicrobial infection in the lungs compared to the WT CLP mice (fig. 5). These data show a critical role of NFATc3 in controlling polymicrobial sepsis.

\section{Discussion}

Severe sepsis is a serious medical condition which often leads to an overwhelming systemic inflammatory response, multiple organ failure and death. The most common elements of sepsis are Gram-negative bacteria and the bacterial endotoxin (LPS), which activate the immune responses resulting in production of proinflammatory immune modulators [1]. The lung, abdominal cavity and primary infections of the bloodstream are the most common sites of infection in bacterial sepsis. There are currently no effective, specific treatments that are based on molecular pathogenesis; thus it is imperative to understand the molecular mechanism mediating the bacterial clearance during sepsis. Macrophages are innate immune effector cells that are involved in generating beneficial inflammation that is necessary for pulmonary host defense [33]. In contrast, dysregulated macrophages can contribute to harmful collateral lung damage due to either exuberant or persistent lung inflammation. Engagement of LPS with TLR4 on the surface of a pulmonary macro-

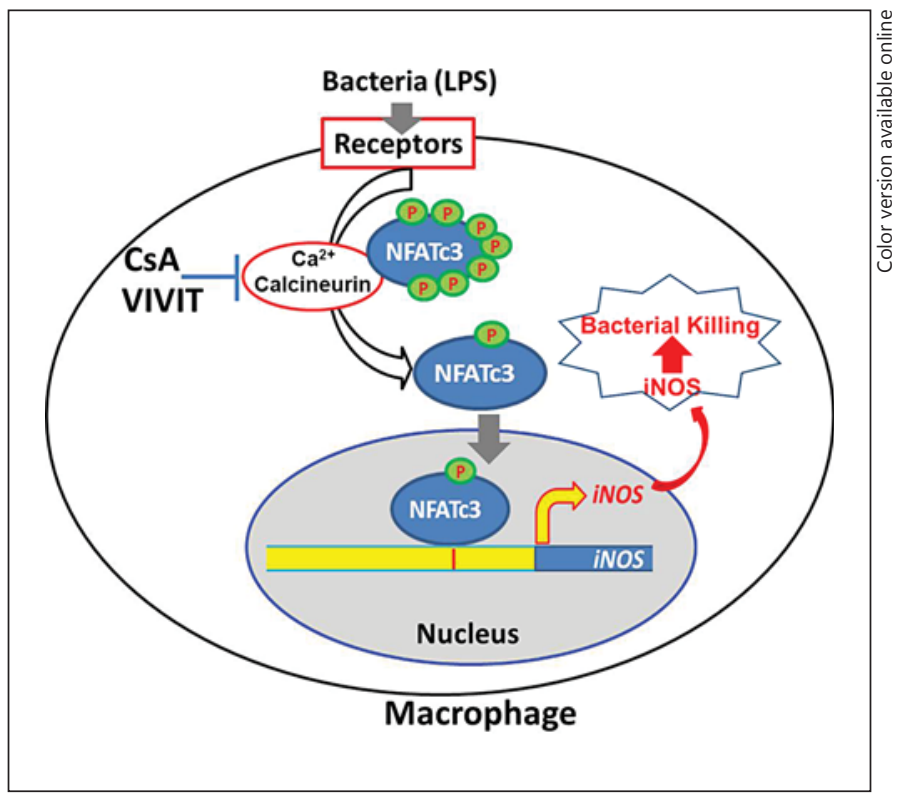

Fig. 6. Transcription factor NFATc3 regulates macrophage bactericidal activity. During the onset of sepsis by bacterial infection, the transcription factor NFATc3 in macrophages is activated by the change in the calcium levels, which results in its translocation to the nucleus, resulting in the transcription of $i N O S$. This results in robust generation of $\mathrm{NO}$ and other reactive oxygen/nitrogen intermediates, resulting in efficient bactericidal activity and thus contributing to the resolution of harmful bacterial infection and inflammation.

phage results in activation of NADPH oxidase by driving a protein-protein interaction among the cytoplasmic and membrane components of NADPH oxidase that results in production of ROS. It is well known that NO, which is a major contributing factor of cellular ROS in macrophages, is produced from iNOS, also known as type II NOS. NO plays important roles in many different aspects of mammalian biology and is an important component of host defense against intracellular pathogens; however, with subsequent formation of peroxynitrite and poly(adenosine diphosphate ribose) it is critically implicated in the pathophysiology of acute lung injury and sepsis [6]. Macrophages are intrinsically more responsive to endotoxin than neutrophils, basophils or eosinophils [34] due to increased expression of TLR4, which can be markedly upregulated by treatment with certain stimuli [35].

Until very recently, NF- $\kappa \mathrm{B}$ proteins were the only Rellike transcription factors known to be involved in the innate immune response in macrophages and other immune cells; however, recent publications have emphasized the role of the transcription factor NFAT in 
macrophages as well $[20,23]$. NFATs play a key role in the development and function of immune cells [16] and were initially identified in modulating $\mathrm{T}$ and $\mathrm{B}$ cell gene expression. However, their role in the modulation of gene expression in macrophages during sepsis has not been explored. KO mice of each NFAT family member exhibit distinct phenotypic defects, possibly due to the different expression profiles of each NFAT among different cell types [36-38]. Recently it has been shown that expression of TLR-induced gene expression, including iNOS, in macrophages is regulated by NFAT5, and this finding is an intriguing phenomenon as it is independent of its osmoregulatory function [39].

Here, we show that NFATc3 actively translocates from the cytosol to the nucleus of LPS-treated macrophages, expression of iNOS in macrophages is mediated by NFATc3 and, more importantly, blocking NFATc3 results in attenuated bactericidal activity. The NFATc3-deficient macrophages cultured from the KO mice had reduced iNOS expression/NO release and exhibited poor bactericidal activity. We tested the biological significance of these in vitro findings in a whole-animal model of severe sepsis due to CLP using NFATc3 KO mice. The KO mice showed decreased bacterial clearance in lungs compared to the control group, indicating that NFATc3 plays a crucial role in containment of bacterial sepsis. Our findings are summarized in the diagram shown in figure 6 . During the onset of sepsis by bacterial infection, the transcription factor NFATc3 in macrophages is activated by the change in calcium levels, which results in its translocation to the nucleus, resulting in the transcriptional upregulation of iNOS. This results in robust generation of $\mathrm{NO}$ and other reactive oxygen/nitrogen intermediates, resulting in efficient bactericidal activity, thus contributing to the resolution of harmful inflammation.

\section{Acknowledgements}

This work was supported by National Institutes of Health Grants R01 HL075557, HL068610 and T32HL082547 and Department of Veterans Affairs Merit Review Grant 5I01BX000108.

\section{Disclosure Statement}

The authors declare that there is no conflict of interests.

\section{References}

1 Cohen J: The immunopathogenesis of sepsis. Nature 2002;420:885-891.

- Fang FC, Vazquez-Torres A: Nitric oxide production by human macrophages: there's no doubt about it. Am J Physiol Lung Cell Mol Physiol 2002;282:L941-L943.

-3 Sadowitz B, Roy S, Gatto LA, Habashi N, Nieman G: Lung injury induced by sepsis: lessons learned from large animal models and future directions for treatment. Expert Rev Anti Infect Ther 2011;9:1169-1178.

4 Toussaint S, Gerlach H: Immunoglobulins in adult sepsis and septic shock. Curr Infect Dis Rep 2012;14:522-529.

5 Pitt BR, St Croix CM: Complex regulation of iNOS in lung. Am J Respir Cell Mol Biol 2002; 26:6-9.

6 MacMicking J, Xie Q-W, Nathan C: Nitric oxide and macrophage function. Annu Rev Immunol 1997; 15:323-350.

-7 Baron RM, Carvajal IM, Liu X, Okabe RO, Fredenburgh LE, Macias AA, Chen Y-H, Ejima K, Layne MD, Perrella MA: Reduction of nitric oxide synthase 2 expression by distamycin A improves survival from endotoxemia. J Immunol 2004;173:4147-4153.

-8 Farley KS, Wang LF, Razavi HM, Law C, Rohan M, McCormack DG, Mehta S: Effects of macrophage inducible nitric oxide synthase in murine septic lung injury. Am J Physiol Lung Cell Mol Physiol 2006;290:L1164-L1172. $\checkmark$ Diefenbach A, Schindler H, Donhauser N, Lorenz E, Laskay T, MacMicking J, Röllinghoff M, Gresser I, Bogdan C: Type 1 interferon (IFN $/ \beta$ ) and type 2 nitric oxide synthase regulate the innate immune response to a protozoan parasite. Immunity 1998;8:77-87.

10 Nathan C, Shiloh MU: Reactive oxygen and nitrogen intermediates in the relationship between mammalian hosts and microbial pathogens. Proc Natl Acad Sci USA 2000;97: 8841-8848.

11 Bogdan C: Nitric oxide and the immune response. Nat Immunol 2001;2:907-916.

-12 D’Alessio FR, Tsushima K, Aggarwal NR, Mock JR, Eto Y, Garibaldi BT, Files DC, Avalos CR, Rodriguez JV, Waickman AT, Reddy SP, Pearse DB, Sidhaye VK, Hassoun PM, Crow MT, King LS: Resolution of experimental lung injury by monocyte-derived inducible nitric oxide synthase. J Immunol 2012;189:2234-2245.

13 Kawai T, Akira S: Toll-like receptors and their crosstalk with other innate receptors in infection and immunity. Immunity 2011;34:637650.

14 Fortin CF, McDonald PP, Fulop T, Lesur O: Sepsis, leukocytes, and nitric oxide (NO): an intricate affair. Shock 2010;33:344-352.

15 Feske S, Rao A, Hogan PG: The Ca2+-calcineurin-NFAT signalling pathway. New Compr Biochem 2007;41:365-401.
16 Hogan PG, Chen L, Nardone J, Rao A: Transcriptional regulation by calcium, calcineurin, and NFAT. Genes Dev 2003;17:22052232.

17 Semenova SB, Kiselev KI, Mozhaeva GN: Low-conductivity calcium channels in the macrophage plasma membrane: activation by inositol-1,4,5-triphosphate. Neurosci Behav Physiol 1999;29:339-345.

18 Lee C, Liu QH, Tomkowicz B, Yi Y, Freedman BD, Collman RG: Macrophage activation through CCR5- and CXCR4-mediated gp120-elicited signaling pathways. J Leukoc Biol 2003;74:676-682.

19 Serafini N, Dahdah A, Barbet G, Demion M, Attout T, Gautier G, Arcos-Fajardo M, Souchet H, Jouvin MH, Vrtovsnik F, Kinet JP, Benhamou M, Monteiro RC, Launay P: The TRPM4 channel controls monocyte and macrophage, but not neutrophil, function for survival in sepsis. J Immunol 2012;189:36893699.

20 Minematsu $\mathrm{H}$, Shin MJ, Celil Aydemir AB, Kim KO, Nizami SA, Chung GJ, Lee FY: Nuclear presence of nuclear factor of activated $\mathrm{T}$ cells (NFAT) c 3 and c4 is required for Tolllike receptor-activated innate inflammatory response of monocytes/macrophages. Cell Signal 2011;23:1785-1793. 
21 Ulrich JD, Kim MS, Houlihan PR, Shutov LP, Mohapatra DP, Strack S, Usachev YM: Distinct activation properties of the nuclear factor of activated T-cells (NFAT) isoforms NFAT3 and NFAT4 in neurons. J Biol Chem 2012;287:37594-37609.

22 Bonnet S, Rochefort G, Sutendra G, Archer SL, Haromy A, Webster L, Hashimoto K, Bonnet SN, Michelakis ED: The nuclear factor of activated $\mathrm{T}$ cells in pulmonary arterial hypertension can be therapeutically targeted. Proc Natl Acad Sci USA 2007;104:1141811423.

23 Elloumi HZ, Maharshak N, Rao KN, Kobayashi T, Ryu HS, Muhlbauer M, Li F, Jobin C, Plevy SE: A cell permeable peptide inhibitor of NFAT inhibits macrophage cytokine expression and ameliorates experimental colitis. PLoS One 2012;7:e34172.

-24 Musiek ES, Brooks JD, Joo M, Brunoldi E, Porta A, Zanoni G, Vidari G, Blackwell TS, Montine TJ, Milne GL, McLaughlin B, Morrow JD: Electrophilic cyclopentenone neuroprostanes are anti-inflammatory mediators formed from the peroxidation of the omega-3 polyunsaturated fatty acid docosahexaenoic acid. J Biol Chem 2008;283:19927-19935.

-25 Sadikot RT, Zeng H, Yull FE, Li B, Cheng DS, Kernodle DS, Jansen ED, Contag CH, Segal BH, Holland SM, Blackwell TS, Christman JW: p47phox deficiency impairs NF-kappa B activation and host defense in Pseudomonas pneumonia. J Immunol 2004;172:1801-1808.
6 Cartharius K, Frech K, Grote K, Klocke B, Haltmeier M, Klingenhoff A, Frisch M, Bayerlein $\mathrm{M}$, Werner T: MatInspector and beyond: promoter analysis based on transcription factor binding sites. Bioinformatics 2005; 21:2933-2942.

27 Campbell PA, Canono BP, Drevets DA: Measurement of bacterial ingestion and killing by macrophages. Curr Protoc Immunol 2001; chapter 14:unit 14.6.

28 Rittirsch D, Huber-Lang MS, Flierl MA, Ward PA: Immunodesign of experimental sepsis by cecal ligation and puncture. Nat Protoc 2009;4:31-36.

29 Liu J, Farmer JD Jr, Lane WS, Friedman J, Weissman I, Schreiber SL: Calcineurin is a common target of cyclophilin-cyclosporin A and FKBP-FK506 complexes. Cell 1991;66: 807-815.

30 Noguchi $\mathrm{H}$, Matsushita M, Okitsu T, Moriwaki A, Tomizawa K, Kang S, Li ST, Kobayashi N, Matsumoto S, Tanaka K, Tanaka N, Matsui H: A new cell-permeable peptide allows successful allogeneic islet transplantation in mice. Nat Med 2004;10:305-309.

31 Xie QW, Kashiwabara Y, Nathan C: Role of transcription factor NF-kappa $\mathrm{B} / \mathrm{Rel}$ in induction of nitric oxide synthase. J Biol Chem 1994;269:4705-4708.

32 Xie Q, Nathan C: The high-output nitric oxide pathway: role and regulation. J Leukoc Biol 1994;56:576-582.

33 Parihar A, Eubank TD, Doseff AI: Monocytes and macrophages regulate immunity through dynamic networks of survival and cell death. J Innate Immun 2010;2:204-215.
34 Sabroe I, Jones EC, Usher LR, Whyte MK, Dower SK: Toll-like receptor (TLR)2 and TLR4 in human peripheral blood granulocytes: a critical role for monocytes in leukocyte lipopolysaccharide responses. J Immunol 2002;168:4701-4710.

35 Mita Y, Dobashi K, Shimizu Y, Nakazawa T, Mori M: Toll-like receptor 2 and 4 surface expressions on human monocytes are modulated by interferon-gamma and macrophage colony-stimulating factor. Immunol Lett 2001;78:97-101.

-36 Abbott KL, Friday BB, Thaloor D, Murphy TJ, Pavlath GK: Activation and cellular localization of the cyclosporine A-sensitive transcription factor NF-AT in skeletal muscle cells. Mol Biol Cell 1998;9:2905-2916.

37 Amasaki Y, Adachi S, Ishida Y, Iwata M, Arai N, Arai K, Miyatake S: A constitutively nuclear form of NFATx shows efficient transactivation activity and induces differentiation of CD4(+)CD8(+) T cells. J Biol Chem 2002;277: 25640-25648.

-38 Obasanjo-Blackshire K, Mesquita R, Jabr RI, Molkentin JD, Hart SL, Marber MS, Xia Y, Heads RJ: Calcineurin regulates NFAT-dependent iNOS expression and protection of cardiomyocytes: co-operation with Src tyrosine kinase. Cardiovasc Res 2006;71:672-683.

39 Buxade M, Lunazzi G, Minguillon J, Iborra S, Berga-Bolanos R, Del Val M, Aramburu J, Lopez-Rodriguez C: Gene expression induced by toll-like receptors in macrophages requires the transcription factor NFAT5. J Exp Med 2012;209:379-393. 